\title{
A note on tensor fields in Hilbert spaces
}

\author{
LEONARDO BILIOTTI ${ }^{1}$, FRANCESCO MERCURI ${ }^{1}$ and DANIEL V. TAUSK ${ }^{2}$ \\ ${ }^{1}$ IMECC - UNICAMP, C.P. 6065, 13083-970 Campinas, SP, Brazil \\ ${ }^{2}$ IME-USP, Cidade Universitária, Rua da Reitoria 109, Butantã, São Paulo, SP, Brazil
}

Manuscript received on December 15, 2001; accepted for publication on February 26, 2001; presented by MANFREDO DO CARMO

\begin{abstract}
We discuss and extend to infinite dimensional Hilbert spaces a well-known tensoriality criterion for linear endomorphisms of the space of smooth vector fields in $\mathbb{R}^{n}$.
\end{abstract}

Key words: Tensor fields, Hilbert spaces.

\section{INTRODUCTION}

A basic fact in classical differential geometry, called the "Fundamental lemma of differential geometry" in Besse (1987), is that an $\mathbb{R}$-linear endomorphism of the space of vector fields in an open set of $\mathbb{R}^{n}$, is a tensor field (of type $(1,1)$ ) if and only if is linear with respect to functions. This is not any more true in infinite dimensions and the aim of this note is to give a contra-example and to introduce a class of endomorphism for which the criterion holds true. Some basic references for infinite dimensional differential geometry are Lang (1995) and Abraham et al. (1988).

\section{TENSOR FIELDS IN HILBERT SPACES}

Let $\mathbb{H}$ be a real Hilbert space, $\mathrm{B}(\mathbb{H})$ be the space of bounded linear endomorphisms of $\mathbb{H}$ and $\Omega \subset \mathbb{H}$ be an open set. We will denote by $\mathcal{H}(\Omega)$ the space of (smooth) vector fields in $\Omega$, i.e., the space of smooth maps $\xi: \Omega \rightarrow \mathbb{H}$; by $\mathcal{F}(\Omega)$ we denote the algebra of smooth real valued functions in $\Omega$. Then $\mathcal{H}(\Omega)$ is a real vector space and an $\mathcal{F}(\Omega)$-module in the obvious way.

We consider a map $A: \Omega \rightarrow \mathrm{B}(\mathbb{H})$ and, for $\xi \in \mathcal{H}(\Omega)$, we define a vector field $\tilde{A}(\xi)$ in $\Omega$ by setting $\tilde{A}(\xi)(x)=A(x) \cdot \xi(x)$. If $\tilde{A}(\xi)$ is smooth for all $\xi \in \mathcal{H}(\Omega)$ then we way that $A$ is weakly 
smooth; in this case $\tilde{A}$ is a $\mathcal{F}(\Omega)$-linear endomorphism of $\mathcal{H}(\Omega)$. Clearly, if $A$ is smooth then it is also weekly smooth and if $\operatorname{dim}(\mathbb{H})<+\infty$ the converse holds.

Now we consider an arbitrary $\mathbb{R}$-linear map $\tilde{A}: \mathcal{H}(\Omega) \rightarrow \mathcal{H}(\Omega)$. Recall that:

1. $\tilde{A}$ is a tensor field (of type $(1,1)$ ) if there exist a weakly smooth map $A: \Omega \rightarrow \mathrm{B}(\mathbb{H})$ such that: $\tilde{A}(\xi)(x)=A(x) \cdot \xi(x)$, for all $x \in \Omega$.

2. $\tilde{A}$ is punctual if, for all $\xi \in \mathcal{H}(\Omega), x \in \Omega, \xi(x)=0$ implies $\tilde{A}(\xi)(x)=0$.

3. $\tilde{A}$ is a zero-order differential operator if $\tilde{A}$ is $\mathcal{F}(\Omega)$-linear.

REMARK 1. The $\mathcal{F}(\Omega)$-linearity implies that $\tilde{A}$ is a differential operator, i.e., $\left.\xi\right|_{U}=0$ implies $\left.\tilde{A}(\xi)\right|_{U}=0$ for all $\xi \in \mathcal{H}(\Omega)$ and for every open subset $U \subset \Omega$.

Conditions (1) and (2) are obviously equivalent and any of them implies condition (3). It is well known and easy to prove that if $\operatorname{dim}(\mathbb{H})=n<+\infty$ then conditions (1), (2) and (3) are all equivalent. We briefly recall the proof of the implication $(3) \Rightarrow(2)$. Let $\left(e_{i}\right)_{i=1}^{n}, n=\operatorname{dim}(\mathbb{H})<+\infty$, be a basis for $\mathbb{H}$ and, given $\xi \in \mathcal{H}(\Omega)$, write $\xi=\sum_{i=1}^{n} \xi_{i} e_{i}$, where each $\xi_{i} \in \mathcal{F}(\Omega)$. The $\mathcal{F}(\Omega)$ linearity of $\tilde{A}$ implies $\tilde{A}(\xi)=\sum_{i=1}^{n} \xi_{i} \tilde{A}\left(e_{i}\right)$ from which condition (2) follows.

The aim of this note is to discuss the relations between conditions (2) and (3) in the case that $\mathbb{H}$ is infinite-dimensional. We start by pointing out that such conditions are no longer equivalent as the following example shows.

EXAMPLE 2. Let $\lambda: \mathrm{B}(\mathbb{H}) \rightarrow \mathbb{H}$ be a continuous non zero linear map whose kernel contains the closed subspace of compact endomorphisms of $\mathbb{H}$. Set:

$$
\tilde{A}(\xi)(x)=\lambda(\mathrm{d} \xi(x)), \quad \xi \in \mathcal{H}(\Omega), x \in \Omega
$$

where $\mathrm{d} \xi(x) \in \mathrm{B}(\mathbb{H})$ denotes the differential of the smooth map $\xi$ at the point $x$. We claim that $\tilde{A}$ is an $\mathcal{F}(\Omega)$-linear operator. Namely, if $f \in \mathcal{F}(\Omega)$ and if $f(x)=0$ for some $x \in \Omega$ then:

$$
\mathrm{d}(f \xi)(x) v=[d f(x) v] \xi(x) .
$$

Hence, $\mathrm{d}(f \xi)(x) \in \mathrm{B}(\mathbb{H})$ has 1-dimensional range and therefore it is a compact operator and $\tilde{A}(f \xi)(x)=0$. Now, in general, if $f \in \mathcal{F}(\Omega)$ and $x \in \Omega$, we have:

$$
\tilde{A}(f \xi)(x)=\tilde{A}[(f-f(x)) \xi+f(x) \xi](x)=f(x) \tilde{A}(\xi)(x),
$$

which proves that $\tilde{A}$ is $\mathcal{F}(\Omega)$-linear. On the other hand $\tilde{A}$ cannot be punctual because if $\xi \in \mathrm{B}(\mathbb{H})$ is not in the kernel of $\lambda$ then $\tilde{A}(\xi)(0)$ is not zero.

If we assume the continuity of the operator $\tilde{A}$ with respect to pointwise convergence in $\mathcal{H}(\Omega)$ then we can proceed in analogy with the finite dimensional case to prove the implication $(3) \Rightarrow(2)$. Such assumption, however, is too strong since in all interesting cases directional derivatives appear in the expression for $\tilde{A}$. Assume, for simplicity, that $\mathbb{H}$ is separable (the general case follow 
substituting sequences for nets). Fix a Hilbert space basis $\left(e_{i}\right)_{i=1}^{+\infty}$ for $\mathbb{H}$ then, for $\xi \in \mathcal{H}(\Omega)$, we have $\xi=\sum_{i=1}^{+\infty} \xi_{i} e_{i}, \xi_{i} \in \mathcal{F}(\Omega)$, and this series converges pointwise together with all its directional derivatives. In fact, the $i$-th coordinate operator $\mathbb{H} \ni \xi \mapsto \xi_{i}=\left\langle\xi, e_{i}\right\rangle \in \mathbb{R}$ is linear and continuous and hence it commutes with directional derivative operators $\frac{\partial}{\partial v}: \mathcal{H}(\Omega) \rightarrow \mathcal{H}(\Omega)$, $v \in \mathbb{H}$.

The above considerations lead to the following:

Definition 3. An operator $\tilde{A}: \mathcal{H}(\Omega) \rightarrow \mathcal{H}(\Omega)$ is weakly $C^{\infty}$-continuous if for every sequence $\left(\xi^{k}\right)_{k \in \mathbb{N}}$ in $\mathcal{H}(\Omega)$ converging pointwise with all its directional derivatives to $\xi \in \mathcal{H}(\Omega)$, the sequence $\left(\tilde{A}\left(\xi^{k}\right)(x)\right)_{k \in \mathbb{N}}$ converges to $\tilde{A}(\xi)(x)$ weakly in $\mathbb{H}$, for all $x \in \Omega$.

THEOREM 4. If $\tilde{A}$ is weakly $C^{\infty}$-continuous, then $(3) \Rightarrow(2)$.

Proof. Let $\xi \in \mathcal{H}(\Omega), x \in \Omega$ and assume $\xi(x)=0$. We write $\xi=\sum_{i=1}^{+\infty} \xi_{i} e_{i}, \xi_{i} \in \mathcal{F}(\Omega)$, where $\left(e_{i}\right)_{i=1}^{+\infty}$ is a Hilbert basis for $\mathbb{H}$. By setting $\xi^{k}=\sum_{i=1}^{k} \xi_{i} e_{i}$ then, as observed above, $\left(\xi^{k}\right)_{k \in \mathbb{N}}$ converges pointwise to $\xi$ together with all its directional derivatives. Since $\tilde{A}$ is weakly $C^{\infty}$ continuous, $\left(\tilde{A}\left(\xi^{k}\right)(x)\right)_{k \in \mathbb{N}}$ converges weakly to $\tilde{A}(\xi)(x)$ in $\mathbb{H}$. Finally, by the $\mathcal{F}(\Omega)$-linearity of $\tilde{A}:$

$$
\tilde{A}\left(\xi^{k}\right)(x)=\sum_{i=1}^{k} \xi_{i}(x) \tilde{A}\left(e_{i}\right)(x)=0, \quad \text { for all } k \in \mathbb{N}
$$

which implies $\tilde{A}(\xi)(x)=0$ and concludes the proof.

We will now discuss the result above in an interesting case. We recall that a linear connection in $\mathbb{H}$ is an $\mathbb{R}$-bilinear map:

$$
\nabla: \mathcal{H}(\Omega) \times \mathcal{H}(\Omega) \ni(X, Y) \longmapsto \nabla_{X} Y \in \mathcal{H}(\Omega)
$$

such that:

- $\nabla$ is $\mathcal{F}(\Omega)$-linear in the first variable;

- for all $X, Y \in \mathcal{H}(\Omega), f \in \mathcal{F}(\Omega)$, the identity:

$$
\nabla_{X}(f Y)=X(f) Y+f \nabla_{X} Y
$$

holds.

An easy modification of Example 2 shows that not all connections are punctual in the first argument. However, if we give a Riemannian metric in $\Omega$ i.e., a smooth map $g: \Omega \rightarrow \mathrm{B}(\mathbb{H})$ such that for all $x \in \Omega, g(x): \mathbb{H} \rightarrow \mathbb{H}$ is self-adjoint and there exists a positive real-valued function $K$ such that $\|g(x) v\| \geq K(x)\|v\|$, we have a special connection, the Levi-Civita connection, uniquely defined by the two extra conditions: 
- $\nabla_{X} Y-\nabla_{Y} X=[X, Y]=d Y(X)-d X(Y)$, for all $X, Y \in \mathcal{H}(\Omega)$;

- $X g(Y, Z)=g\left(\nabla_{X} Y, Z\right)+g\left(Y, \nabla_{X} Z\right)$, for all $X, Y, Z \in \mathcal{H}(\Omega)$,

where $g(X, Y)(x)=\langle g(x) X, Y\rangle$. Observe that, for all $x \in \Omega, g(x)$ defines an inner product in $\mathbb{H}$ compatible with its topology. The Levi-Civita connection can be explicitly described by the Koszul formula:

$$
\begin{array}{rl}
2 g\left(\nabla_{X} Y, Z\right)=X & g(Y, Z)+Y g(Z, X)-Z g(X, Y) \\
& +g([X, Y], Z)+g([Z, X], Y)-g([Y, Z], X) .
\end{array}
$$

It follows from the properties of the Lie brackets, that the expression on the righthand side of (1) is $\mathcal{F}(\Omega)$-linear in $X$ and in $Z$. Moreover, since such expression involves only partial derivatives, it is also weakly $C^{\infty}$-continuous on $X$ and $Z$. Hence, Theorem 4 implies that the righthand side of (1) is indeed punctual in $X$ and $Z$, so that $\nabla_{X} Y$ is well-defined by (1) and it is punctual in $X$.

In general, given a connection $\nabla$ we can write $\nabla_{X} Y=d Y(X)+\Gamma(X, Y)$, where $\Gamma$ : $\mathcal{H}(\Omega) \times \mathcal{H}(\Omega) \rightarrow \mathcal{H}(\Omega)$ is $\mathcal{F}(\Omega)$-bilinear. Classically, $\Gamma$ is known as the Christoffel symbol of the connection. Using essentially the same argument as above, we conclude that the Christoffel symbol of the Levi-Civita connection is punctual in both arguments.

Also we can consider the Riemannian curvature:

$$
R(X, Y) Z=\nabla_{X} \nabla_{Y} Z-\nabla_{Y} \nabla_{X} Z-\nabla_{[X, Y]} Z
$$

where $\nabla$ denotes the Levi-Civita connection. $R$ is $\mathcal{F}(\Omega)$-linear in all arguments. Again, the fact that $R$ is weakly $C^{\infty}$-continuous implies, by Theorem 4 , that $R$ is punctual in all its variables.

\section{ACKNOWLEDGMENT}

The authors thank CNPq and FAPESP for partial support.

\section{RESUMO}

Discutimos e estendemos para espaços de Hilbert um critério de tensorialidade para endomorfismos do espaço dos campos vetoriais em $\mathbb{R}^{n}$.

Palavras-chave: campos tensoriais, espaços de Hilbert.

\section{REFERENCES}

Abraham R, Marsden JE and Ratiu T. 1988. Manifolds, Tensor Analysis and Applications, second edition, Applied Mathematical Science, Springer Verlag, New York, 1988.

Besse A. 1987. Einstein Manifolds, Springer-Verlag, Berlin Heidelberg.

LANG S. 1995. Differential and Riemannian Manifolds, third edition, Graduate text in Mathematics, 160, Springer-Verlag, New York, 1995. 